\title{
Strong convergence of iterative algorithms with variable coefficients for asymptotically strict pseudocontractive mappings in the intermediate sense and monotone mappings
}

\section{Ci-Shui Ge}

Correspondence: gecishui@sohu. com

Department of Mathematics and Physics, Anhui University of

Architecture, Jinzhai Road, Hefei, Anhui, People's Republic of China

\begin{abstract}
In this article, we propose some iterative algorithms with variable coefficients for finding a common element of the set of fixed points of a uniformly continuous asymptotically $\kappa$-strict pseudocon-tractive mapping in the intermediate sense and the set of solutions of the variational inequality problem for a monotone, Lipschitzcontinuous mapping. Some strong convergence theorems of these iterative algorithms are obtained without some boundedness assumptions and without some convergence condition. The results of the article improve and extend the recent results of Ceng and Yao, Nadezhkina and Takahashi, and several others.

Mathematics Subject Classification (2000): 47H09; 47J2O.

Keywords: fixed point, variational inequality, asymptotically strict pseudocontractive mapping in the intermediate sense, monotone mapping, variable coefficient method
\end{abstract}

\section{Introduction}

Let $H$ be a real Hilbert space with inner product $\langle.,$.$\rangle and norm \|\cdot\|$, respectively. Let $C$ a nonempty closed convex subset of $H$ and let $P_{C}$ be the metric project from $H$ onto C. $F(S)=\{x \in C: S x=x\}$ denotes the set of fixed points of a self-mapping $S$ on a set $C$.

A mapping $A$ of $C$ into $H$ is called monotone if

$$
\langle A x-A y, x-y\rangle \geq 0, \quad \forall x, y \in C .
$$

A mapping $A$ of $C$ into $H$ is said to be $L$-Lipschitz continuous if there exists a positive constant $L$ such that

$$
\|A x-A y\| \leq L\|x-y\|, \quad \forall x, y \in C .
$$

Let the mapping $A$ from $C$ to $H$ be monotone and Lipschitz continuous. The variational inequality problem (for short, $V I(C, A)$ ) is to find a $u \in C$ such that

$$
\langle A u, v-u\rangle \geq 0, \quad \forall v \in C .
$$

The set of solutions of the $\operatorname{VI}(C, A)$ is denoted $\Omega$. A mapping $A$ of $C$ into $H$ is said to be $\alpha$-inverse strongly monotone if there exists a positive constant $\alpha$ such that

(c) 2012 Ge; licensee Springer. This is an Open Access article distributed under the terms of the Creative Commons Attribution License (http://creativecommons.org/licenses/by/2.0), which permits unrestricted use, distribution, and reproduction in any medium, provided the original work is properly cited. 


$$
\langle A x-A y, x-y\rangle \geq \alpha\|A x-A y\|^{2}, \quad \forall x, y \in C .
$$

It is obvious that if $A$ is $\alpha$-inverse-strongly monotone, then $A$ is monotone and Lipschitz continuous. Recall that a mapping $S: C \rightarrow C$ is called to be nonexpansive if

$$
\|S x-S y\| \leq\|x-y\|, \quad \forall x, y \in C .
$$

A mapping $S: C \rightarrow C$ is called to be asymptotically nonexpansive [1], if there exists a sequence $\left\{k_{n}\right\} \subset[1, \infty)$ with $k_{n} \rightarrow 1$ as $n \rightarrow \infty$ such that

$$
\left\|S^{n} x-S^{n} y\right\| \leq k_{n}\|x-y\|, \quad \text { for all } x, y \in C, \quad \text { and all integers } n \geq 1 .
$$

$S: C \rightarrow C$ is called to be asymptotically nonexpansive in the intermediate sense [2], if it is continuous and the following inequality holds:

$$
\limsup _{n \rightarrow \infty} \sup _{x, y \in C}\left(\left\|S^{n} x-S^{n} y\right\|-\|x-y\|\right) \leq 0 .
$$

In fact, we see that (1.1) is equivalent to

$$
\left\|S^{n} x-S^{n} y\right\|^{2} \leq\|x-y\|^{2}+c_{n}, \quad \forall n \geq 1, x, y \in C,
$$

where $c_{n} \in[0, \infty)$ with $c_{n} \rightarrow 0$ as $n \rightarrow \infty$. $S$ is called to be an asymptotically $\kappa$-strict pseudocontractive mapping with sequence $\left\{\gamma_{n}\right\}$ [3], if there exist a constant $\kappa \in[0,1)$ and a sequence $\left\{\gamma_{n}\right\} \subset[0, \infty)$ with $\gamma_{n} \rightarrow 0$ as $n \rightarrow \infty$ such that

$$
\left\|S^{n} x-S^{n} y\right\|^{2} \leq\left(1+\gamma_{n}\right)\|x-\gamma\|^{2}+\kappa\left\|\left(I-S^{n}\right) x-\left(I-S^{n}\right) y\right\|^{2}
$$

for all $x, y \in C$ and all integers $n \geq 1$. It is very clear that, in a real Hilbert space $H$, (1.3) is equivalent to

$$
\left\langle S^{n} x-S^{n} y, x-y\right\rangle \leq\left(1+\frac{\gamma_{n}}{2}\right)\|x-y\|^{2}-\frac{1-\kappa}{2}\left\|\left(I-S^{n}\right) x-\left(I-S^{n}\right) y\right\|^{2} .
$$

Recall that $S$ is called to be an asymptotically $\kappa$-strict pseudocontraction in the intermediate sense with sequence $\left\{\gamma_{n}\right\}$ [4], if

$$
\limsup _{n \rightarrow \infty} \sup _{x, y \in C}\left\{\left\|S^{n} x-S^{n} y\right\|^{2}-\left(1+\gamma_{n}\right)\|x-y\|^{2}-\kappa\left\|\left(I-S^{n}\right) x-\left(I-S^{n}\right) y\right\|^{2}\right\} \leq 0,
$$

where $\kappa \in[0,1)$ and $\gamma_{n} \in[0, \infty)$ such that $\gamma_{n} \rightarrow 0$ as $n \rightarrow \infty$. In fact, (1.4) is reduced to the following:

$$
\left\|S^{n} x-S^{n} y\right\|^{2} \leq\left(1+\gamma_{n}\right)\|x-y\|^{2}+\kappa\left\|\left(I-S^{n}\right) x-\left(I-S^{n}\right) y\right\|^{2}+c_{n}, \forall x, y \in C,
$$

where $c_{n} \in[0, \infty)$ with $c_{n} \rightarrow 0$ as $n \rightarrow \infty$.

Recently, by combining Korpelevich's extragradient method [5] with TakahashiToyoda's iterative algorithm [6], Nadezhkina and Takahashi [7] introduced the following iterative scheme for finding a common element of $F(S) \cap \Omega$, the intersection of the set of fixed points of a nonexpansive mapping and the set of solutions of the variational inequality problem for a monotone, Lipschitz-continuous mapping and proved its weak convergence.

Theorem A (See [[7], Theorem 3.1]). Let $C$ be a nonempty closed convex subset of a Hilbert space $H$. Let $A: C \rightarrow H$ be a monotone, L-Lipschitz continuous mapping and let $S: C \rightarrow C$ be a nonexpansive mapping such that $F(S) \cap \Omega \neq \emptyset$. Let $\left\{x_{n}\right\}$ and $\left\{y_{n}\right\}$ be 
the sequences generated by

$$
\left\{\begin{array}{l}
x_{0}=x \in C \text { chosen arbitrary, } \\
y_{n}=P_{C}\left(x_{n}-\lambda_{n} A x_{n}\right), \\
x_{n+1}=\alpha_{n} x_{n}+\left(1-\alpha_{n}\right) S P_{C}\left(x_{n}-\lambda_{n} A y_{n}\right), \quad \forall n \geq 0,
\end{array}\right.
$$

where $\lambda_{n} \subset[a, b]$ for some $a, b \in(0,1 / L)$ and $\alpha_{n} \subset[c, d]$ for some $c, d \in(0,1)$. Then the sequences $\left\{x_{n}\right\},\left\{y_{n}\right\}$ converge weakly to the same point $z \in F(S) \cap \Omega$, where $z=$ $\lim _{n \rightarrow \infty} P_{F(S) \cap \Omega} x_{n}$.

Inspired by Nadezhkina and Takahashi [[7], Theorem 3.1], and Zeng and Yao [8] introduced the following iterative process for finding an element of $F(S) \cap \Omega$ and established the following strong convergence theorem.

Theorem B (See [[8], Theorem 3.1]). Let C be a nonempty closed convex subset of a Hilbert space $H$. Let $A: C \rightarrow H$ be a monotone, L-Lipschitz continuous mapping and let $S: C \rightarrow C$ be a nonexpansive such that $F(S) \cap \Omega \neq \emptyset$. Let $\left\{x_{n}\right\}$ and $\left\{y_{n}\right\}$ be the sequences generated by any given $x_{0} \in C$ and

$$
\left\{\begin{array}{l}
y_{n}=P_{C}\left(x_{n}-\lambda_{n} A x_{n}\right), \\
x_{n+1}=\alpha_{n} x_{0}+\left(1-\alpha_{n}\right) S P_{C}\left(x_{n}-\lambda_{n} A y_{n}\right),
\end{array}\right.
$$

for every $n \geq 0$, where $\left\{\lambda_{n}\right\}$ and $\left\{\alpha_{n}\right\}$ satisfy the conditions:

(a) $\left\{\lambda_{n} L\right\} \subset(0,1-\delta)$ for some $\delta \in(0,1)$

(b) $\left\{\alpha_{n}\right\} \subset(0,1), \quad \sum_{n=0}^{\infty} \alpha_{n}=\infty, \quad \lim _{n \rightarrow \infty} \alpha_{n}=0$,

Then the sequences $\left\{x_{n}\right\},\left\{y_{n}\right\}$ converge strongly to the same point $P_{F(S) \cap \Omega}\left(x_{0}\right)$, provided $\lim _{n \rightarrow \infty}\left\|x_{n}-x_{n+1}\right\|=0$.

Further, utilizing the combination of hybrid-type method and extragradient-type method, Nadezhkina and Takahashi [9] introduced the following iterative scheme for finding a common element of $F(S) \cap \Omega$, and proved the following strong convergence theorem.

Theorem C (See [[9], Theorem 3.1] or [[10], Theorem NT2]). Let C be a nonempty closed convex subset of a Hilbert space H. Let $A: C \rightarrow H$ be a monotone, L-Lipschitz continuous mapping and let $S: C \rightarrow C$ be a nonexpansive mapping such that $F(S) \cap \Omega \neq \emptyset$. Let $\left\{x_{n}\right\},\left\{y_{n}\right\}$, and $\left\{z_{n}\right\}$ be the sequences generated by

$$
\left\{\begin{array}{l}
x_{0}=x \in C \text { chosen arbitrary, } \\
y_{n}=P_{C}\left(x_{n}-\lambda_{n} A x_{n}\right) \\
z_{n}=\alpha_{n} x_{n}+\left(1-\alpha_{n}\right) S P_{C}\left(x_{n}-\lambda_{n} A y_{n}\right) \\
C_{n}=\left\{z \in C:\left\|z_{n}-z\right\| \leq\left\|x_{n}-z\right\|\right\} \\
Q_{n}=\left\{z \in C:\left\langle x_{n}-z, x-x_{n}\right\rangle \geq 0\right\} \\
x_{n+1}=P_{C_{n} \cap Q_{n}} x, \quad \forall n \geq 0
\end{array}\right.
$$

where $\lambda_{n} \subset[a, b]$ for some $a, b \in(0,1 / L)$ and $\alpha_{n} \subset[0, c]$ for some $c \in[0,1)$. Then the sequences $\left\{x_{n}\right\},\left\{y_{n}\right\}$, and $\left\{z_{n}\right\}$ converge strongly to the same point $q=P_{F(S) \cap \Omega} x$.

Very recently, considering a nonexpansive nonself mapping, Buong [11] introduced a new iteration method based on the hybrid-type method and extragradient-type method and proved its strong convergence. Considering a finite family of $\kappa$-strict pseudocontractive mappings, Ceng et al. [12] proposed parallel extragradient and cyclic-extragradient algorithms, and derived the weak convergence of these algorithms. 
Most recently, considering a uniformly continuous asymptotically $\kappa$-strict pseudocon-tractive mapping in the intermediate sense, Ceng and Yao [10] proposed a modified hybrid Mann iterative scheme with perturbed mapping based on the hybrid-type method and extragradient-type method, and established the following interesting result with the help of some boundedness assumptions

Theorem D (See [[10], Theorem 3.1]). Let $C$ be a nonempty closed convex subset of a real Hilbert space $H$. Let $A: C \rightarrow H$ be a monotone, L-Lipschitz continuous mapping with $(I-A)(C) \subset C$ and let $S: C \rightarrow C$ be a uniformly continuous asymptotically $\kappa$-strict pseudocontractive mapping in the intermediate sense with sequence $\left\{\gamma_{n}\right\}$ such that $F$ $(S) \cap \Omega$ is nonempty and bounded. Let $\left\{x_{n}\right\},\left\{y_{n}\right\}$, and $\left\{z_{n}\right\}$ be the sequences generated by

$$
\left\{\begin{array}{l}
x_{1}=x \in C \text { chosen arbitrary, } \\
y_{n}=\left(1-\mu_{n}\right) x_{n}+\mu_{n}\left(S^{n} x_{n}-\lambda_{n} A\left(S^{n} x_{n}\right)\right) \\
t_{n}=P_{C}\left(y_{n}-\lambda_{n} A y_{n}\right) \\
z_{n}=\left(1-\alpha_{n}-\beta_{n}\right) x_{n}+\alpha_{n} t_{n}+\beta_{n} S^{n} t_{n} \\
C_{n}=\left\{z \in C:\left\|z_{n}-z\right\|^{2} \leq\left\|x_{n}-z\right\|^{2}+\theta_{n}\right\} \\
Q_{n}=\left\{z \in C:\left\langle x_{n}-z, x-x_{n}\right\rangle \geq 0\right\} \\
x_{n+1}=P_{C_{n} \cap Q_{n}} x, \quad \forall n \geq 1
\end{array}\right.
$$

where $\theta_{n}=\mu_{n} \kappa\left\|x_{n}-S^{n} x_{n}\right\|^{2}+2 \gamma_{n} \Delta_{n}+2 c_{n}+\lambda_{n}^{2}\left(\left\|A\left(S^{n} x_{n}\right)\right\|^{2}+\left\|A y_{n}\right\|^{2}\right)$ and

$$
\Delta_{n}=\max \left\{\sup _{p \in F(S) \cap \Omega}\left\|x_{n}-p\right\|^{2}, \sup _{p \in F(S) \cap \Omega}\left\|t_{n}-p\right\|^{2}\right\}<\infty .
$$

Assume that $\left\{\lambda_{n}\right\}$ is a sequence in $(0,1)$ with $\lim _{n \rightarrow \infty} \lambda_{n}=0$, and $\left\{\alpha_{n}\right\},\left\{\beta_{n}\right\}$, and $\left\{\mu_{n}\right\}$ are three sequences in $[0,1]$ satisfying the conditions:

(i) $\alpha_{n}+\beta_{n} \leq 1$ for all $n \geq 1$;

(ii) $\kappa \leq \alpha_{n}$ and $\lim _{n \rightarrow \infty} \mu_{n}=0$;

(iii) $\liminf _{n \rightarrow \infty} \beta_{n}>0$;

Then the sequences $\left\{x_{n}\right\},\left\{y_{n}\right\}$, and $\left\{z_{n}\right\}$ converge strongly to the same point $q=P_{F(S) \cap \Omega}$ $x$ if and only if $\liminf _{n \rightarrow \infty}\left\langle A x_{n}, y-x_{n}\right\rangle \geq 0$ for all $y \in C$.

The following problems arise naturally then: (1) Can we relax the convergence condition $\liminf _{n \rightarrow \infty}\left\langle A x_{n}, y-x_{n}\right\rangle \geq 0$ for all $y \in C$ in Theorem D by proposing some new algorithm. (2) Can we relax the boundedness assumptions that the intersection $F(S) \cap \Omega$ and the sequence $\left\{\Delta_{n}\right\}$ in Theorem D are both bounded. Actually, in many cases, these assumptions and conditions are not easy to examine in advance. Hence, they are interesting and important problems.

In order to solve these problems, motivated and inspired by Ceng and Yao [10], Nadezhkina and Takahashi [9], and Ge et al. [13], we introduce some new algorithms with variable coefficients based on the hybrid-type method and extragradient-type method for finding a common element of the set of fixed points of a uniformly continuous asymptotically $\kappa$-strict pseudocontractive mapping in the intermediate sense and the set of solutions of the variational inequality problem for a monotone, Lipschitzcontinuous mapping in real Hilbert spaces. Our results improve and extend the 
corresponding results of Ceng and Yao [10], Nadezhkina and Takahashi [9], and several others.

\section{Preliminaries}

Throughout this article,

- $x_{n} \rightarrow x$ means that $\left\{x_{n}\right\}$ converges strongly to $x$.

- $F(S)=\{x \in C: S x=x\}$ denotes the set of fixed points of a self-mapping $S$ on a set $C$.

- $B_{r}\left(x_{1}\right):=\left\{x \in H:\left\|x-x_{1}\right\| \leq r\right\}$.

- $\mathbb{N}$ is the set of positive integers.

For every point $x \in H$, there exists a unique nearest point in $C$, denoted by $P_{C} x$, such that

$$
\left\|x-P_{C} x\right\| \leq\|x-y\|, \quad \forall y \in C .
$$

$P_{C}$ is called the metric projection of $H$ onto $C$. We know that $P_{C}$ is a nonexpansive mapping from $H$ onto $C$. Recall that the inequality holds

$$
\left\langle x-P_{C} x, P_{C} x-y\right\rangle \geq 0, \quad \forall x \in H, y \in C .
$$

Moreover, it is easy to see that it is equivalent to

$$
\left\|P_{C} x-P_{C} y\right\|^{2} \leq\left\langle P_{C} x-P_{C} y, x-y\right\rangle, \quad \forall x, y \in H ;
$$

It is also equivalent to

$$
\|x-y\|^{2} \geq\left\|x-P_{C} x\right\|^{2}+\left\|y-P_{C} x\right\|^{2}, \quad \forall x \in H, y \in C .
$$

Lemma 2.1. [14]. Let $C$ be a nonempty closed convex subsets of a real Hilbert space $H$. Given $x \in H$ and $y \in C$. Then $y=P_{C} x$ if and only if there holds the inequality

$$
\langle x-y, y-z\rangle \geq 0, \quad \forall z \in C .
$$

Lemma 2.2. [10]. Let $A: C \rightarrow H$ be a monotone mapping. In the context of the variational inequality problem the characterization of projection (2.1) implies

$$
u \in \Omega \Leftrightarrow u=P_{C}(u-\lambda A u), \quad \forall \lambda>0 .
$$

Lemma 2.3. [15]. Let $C$ be a nonempty closed convex subsets of a real Hilbert space $H$. Given $x, y, z \in H$ and given also a real number a, the set

$$
\left\{v \in C:\|y-v\|^{2} \leq\|x-v\|^{2}+\langle z, v\rangle+a\right\}
$$

is convex and closed.

Lemma 2.4. [16]. Let $H$ be a real Hilbert space. Then for all $x, y, z \in H$ and all $\alpha, \beta$, $\gamma \in[0,1]$ with $\alpha+\beta+\gamma=1$, we have

$$
\|\alpha x+\beta \gamma+\gamma z\|^{2}=\alpha\|x\|^{2}+\beta\|\gamma\|^{2}+\gamma\|z\|^{2}-\alpha \beta\|x-\gamma\|^{2}-\alpha \gamma\|x-z\|^{2}-\beta \gamma\|y-z\|^{2} .
$$

Lemma 2.5. [4]. Let $C$ be a nonempty closed convex subsets of a real Hilbert space $H$. and $S: C \rightarrow C$ be an asymptotically $\kappa$-strict pseudocontraction in the intermediate sense with sequence $\left\{\gamma_{n}\right\}$. Then 


$$
\left\|S^{n} x-S^{n} y\right\| \leq \frac{1}{1-\kappa}\left(\kappa\|x-y\|+\sqrt{\left(1+(1-\kappa) \gamma_{n}\right)\|x-y\|^{2}+(1-\kappa) c_{n}}\right)
$$

for all $x, y \in C$ and $n \geq 1$.

By Ibaraki et al. [[17], Theorem 4.1], we have the following lemma.

Lemma 2.6. Let $\left\{K_{n}\right\}$ be a sequence of nonempty closed convex subsets of a real Hilbert space $H$ such that $K_{n+1} \subset K_{n}$ for each $n \in \mathbb{N}$. If $K^{*}=\cap_{n=0}^{\infty} K_{n}$ is nonempty, then for each $x \in H,\left\{P_{K_{n}} x\right\}$ converges strongly to $P_{K^{*} x}$.

A set-valued mapping $T: H \rightarrow 2^{H}$ is called monotone if for all $x, y \in H, f \in T x$ and $g \in T y$ imply $\langle x-y, f-g\rangle \geq 0$. A monotone mapping $T: H \rightarrow 2^{H}$ is maximal if its graph $G(T)$ is not properly contained in the graph of any other monotone mapping. It is known that a monotone mapping $T$ is maximal if and only if for $(x, f) \in H \times H$,

$\langle x-y, f-g\rangle \geq 0$ for all $(y, g) \in G(T)$ implies $f \in T x$. Let $A: C \rightarrow H$ be a monotone and Lipschitz continuous mapping and let $N_{C} v$ be the normal cone to $C$ at $v \in C$, i.e., $N_{C}=\{w \in H:(v-u, w) \geq 0, \forall u \in C\}$. Define

$$
T v= \begin{cases}A v+N_{C} v, & \text { if } v \in C, \\ \varnothing, & \text { if } v \notin C,\end{cases}
$$

It is known that in this case $T$ is maximal monotone, and $0 \in T v$ if and only if $v \in$ $\Omega$, see [18].

\section{Results and proofs}

Theorem 3.1. Let $C$ be a nonempty closed convex subset of a real Hilbert space $H$. Let $A: C \rightarrow H$ be a monotone, L-Lipschitz continuous mapping and let $S: C \rightarrow C$ be a uniformly continuous asymptotically $\kappa$-strict pseudocontractive mapping in the intermediate sense with sequence $\left\{\gamma_{n}\right\}$ such that $F(S) \cap \Omega$ is nonempty. Let $\left\{x_{n}\right\},\left\{y_{n}\right\}$, and $\left\{z_{n}\right\}$ be the sequences generated by the following algorithm with variable coefficients

$$
\left\{\begin{array}{l}
x_{1} \in C \text { chosen arbitrary, } \\
y_{n}=P_{C}\left(x_{n}-\lambda_{n} A x_{n}\right), \\
t_{n}=P_{C}\left(x_{n}-\lambda_{n} A y_{n}\right) \\
z_{n}=\left(1-\alpha_{n}-\hat{\beta}_{n}\right) x_{n}+\alpha_{n} t_{n}+\hat{\beta}_{n} S^{n} t_{n} \\
C_{0}=C \\
C_{n}=\left\{z \in C_{n-1}:\left\|z_{n}-z\right\|^{2} \leq\left\|x_{n}-z\right\|^{2}-\left(\alpha_{n}-\kappa\right) \hat{\beta}_{n}\left\|t_{n}-S^{n} t_{n}\right\|^{2}+\theta_{n}\right\} \\
x_{n+1}=P_{C_{n}} x_{1}
\end{array}\right.
$$

for every $n \in \mathbb{N}$ where $\hat{\beta}_{n}=\frac{\beta_{n}}{1+\left\|x_{n}-x_{1}\right\|^{2}}, \quad \theta_{n}=\beta_{n}\left(2 \gamma_{n}\left(1+r_{0}^{2}\right)+c_{n}\right), \quad\left\{\alpha_{n}\right\} \subset(a, 1),\left\{\beta_{n}\right\} \subset(b, 1-a)$ and $\left\{\lambda_{n}\right\} \subset$ $(b / L,(1-a) / L)$ for some $a \in(\kappa, 1)$ and some $b \in(0,1-a)$, and $c_{n}$ is as in (1.5), the positive real number $r_{0}$ is chosen so that $B_{r_{0}}\left(x_{1}\right) \cap F(S) \cap \Omega \neq \emptyset$. Then the sequences $\left\{x_{n}\right\},\left\{y_{n}\right\}$, and $\left\{z_{n}\right\}$ converge strongly to a point of $F(S) \cap \Omega$.

Proof. We divide the proof into seven steps.

Step 1. We claim $C_{n}$ is nonempty, convex, and closed for each $n \in \mathbb{N}$.

Indeed, by the assumption, $C_{0}$, i.e., $C$ is nonempty, convex, and closed. From the definition of $C_{n}$ and Lemma 2.3, it is easy to see that $C_{n}$ is convex and closed for each $n \in \mathbb{N}$. We, therefore, only need to prove that $C_{n}$ is nonempty for each $n \in \mathbb{N}$.

Indeed, let $p \in B_{r_{0}}\left(x_{1}\right) \cap F(S) \cap \Omega$ be a arbitrary element. Putting $x=x_{n}-\lambda_{n} A y_{n}$ and $y=p$ in (2.2), we have 


$$
\begin{aligned}
\left\|t_{n}-p\right\|^{2} \leq & \left\|x_{n}-\lambda_{n} A y_{n}-p\right\|^{2}-\left\|x_{n}-\lambda_{n} A y_{n}-t_{n}\right\|^{2} \\
= & \left\|x_{n}-p\right\|^{2}-\left\|x_{n}-t_{n}\right\|^{2}+2 \lambda_{n}\left\langle A y_{n}, p-t_{n}\right\rangle \\
= & \left\|x_{n}-p\right\|^{2}-\left\|x_{n}-t_{n}\right\|^{2}+2 \lambda_{n}\left(\left\langle A y_{n}-A p, p-y_{n}\right\rangle+\left\langle A p, p-y_{n}\right\rangle\right) \\
& \quad+2 \lambda_{n}\left\langle A y_{n}, y_{n}-t_{n}\right\rangle
\end{aligned}
$$

Since $A: C \rightarrow H$ is a monotone mapping and $p \in \Omega=V I(C, A)$, further, we have

$$
\begin{aligned}
\left\|t_{n}-p\right\|^{2} & \leq\left\|x_{n}-p\right\|^{2}-\left\|x_{n}-t_{n}\right\|^{2}+2 \lambda_{n}\left\langle A y_{n}, y_{n}-t_{n}\right\rangle \\
& =\left\|x_{n}-p\right\|^{2}-\left\|x_{n}-y_{n}\right\|^{2}-2\left\langle x_{n}-y_{n}, y_{n}-t_{n}\right\rangle-\left\|y_{n}-t_{n}\right\|^{2}+2 \lambda_{n}\left\langle A y_{n}, y_{n}-t_{n}\right\rangle \\
& =\left\|x_{n}-p\right\|^{2}-\left\|x_{n}-y_{n}\right\|^{2}-\left\|y_{n}-t_{n}\right\|^{2}+2\left\langle x_{n}-\lambda_{n} A y_{n}-y_{n}, t_{n}-y_{n}\right\rangle
\end{aligned}
$$

Since $y_{n}=P_{C}\left(x_{n}-\lambda_{n} A x_{n}\right)$ and $A$ is $L$-Lipschitz continuous, we have

$$
\begin{aligned}
& \left\langle x_{n}-\lambda_{n} A y_{n}-y_{n}, t_{n}-y_{n}\right\rangle \\
= & \left\langle x_{n}-\lambda_{n} A x_{n}-y_{n}, t_{n}-y_{n}\right\rangle+\lambda_{n}\left\langle A x_{n}-A y_{n}, t_{n}-y_{n}\right\rangle \\
\leq & \lambda_{n}\left\langle A x_{n}-A y_{n}, t_{n}-y_{n}\right\rangle \\
\leq & \lambda_{n} L\left\|x_{n}-y_{n}\right\|\left\|t_{n}-y_{n}\right\| .
\end{aligned}
$$

So, it follows from (3.2), (3.3) and $\left\{\lambda_{n}\right\} \subset(b / L,(1-a) / L)$, we obtain

$$
\begin{aligned}
\left\|t_{n}-p\right\|^{2} & \leq\left\|x_{n}-p\right\|^{2}-\left\|x_{n}-y_{n}\right\|^{2}-\left\|y_{n}-t_{n}\right\|^{2}+2 \lambda_{n} L\left\|x_{n}-y_{n}\right\|\left\|t_{n}-y_{n}\right\| \\
& \leq\left\|x_{n}-p\right\|^{2}-\left\|x_{n}-y_{n}\right\|^{2}-\left\|y_{n}-t_{n}\right\|^{2}+\lambda_{n}^{2} L^{2}\left\|x_{n}-y_{n}\right\|^{2}+\left\|t_{n}-y_{n}\right\|^{2} \\
& =\left\|x_{n}-p\right\|^{2}+\left(\lambda_{n}^{2} L^{2}-1\right)\left\|x_{n}-y_{n}\right\|^{2} \\
& \leq\left\|x_{n}-p\right\|^{2} .
\end{aligned}
$$

Since $S: C \rightarrow C$ be an asymptotically $\kappa$-strict pseudocontractive mapping in the intermediate sense with sequences $\left\{\gamma_{n}\right\}$, by the definition, we have

$$
\left\|S^{n} t_{n}-p\right\|^{2} \leq\left(1+\gamma_{n}\right)\left\|t_{n}-p\right\|^{2}+\kappa\left\|t_{n}-S^{n} t_{n}\right\|^{2}+c_{n}, \forall x, y \in C
$$

where $c_{n} \in[0, \infty)$ with $c_{n} \rightarrow 0$ as $n \rightarrow \infty$. So, from $z_{n}=\left(1-\alpha_{n}-\hat{\beta}_{n}\right) x_{n}+\alpha_{n} t_{n}+\hat{\beta}_{n} S^{n} t_{n}$, (3.5), (3.6) and Lemma 2.4, we deduce that

$$
\begin{aligned}
\left\|z_{n}-p\right\|^{2}= & \left\|\left(1-\alpha_{n}-\hat{\beta}_{n}\right)\left(x_{n}-p\right)+\alpha_{n}\left(t_{n}-p\right)+\hat{\beta}_{n}\left(S^{n} t_{n}-p\right)\right\|^{2} \\
\leq & \left(1-\alpha_{n}-\hat{\beta}_{n}\right)\left\|x_{n}-p\right\|^{2}+\alpha_{n}\left\|t_{n}-p\right\|^{2}+\hat{\beta}_{n}\left\|S^{n} t_{n}-p\right\|^{2} \\
& \quad-\alpha_{n} \hat{\beta}_{n}\left\|t_{n}-S^{n} t_{n}\right\|^{2} \\
\leq & \left(1-\alpha_{n}-\hat{\beta}_{n}\right)\left\|x_{n}-p\right\|^{2}+\alpha_{n}\left\|t_{n}-p\right\|^{2} \\
& \quad+\hat{\beta}_{n}\left(\left(1+\gamma_{n}\right)\left\|t_{n}-p\right\|^{2}+\kappa\left\|t_{n}-S^{n} t_{n}\right\|^{2}+c_{n}\right)-\alpha_{n} \hat{\beta}_{n}\left\|t_{n}-S^{n} t_{n}\right\|^{2} \\
= & \left(1-\alpha_{n}-\hat{\beta}_{n}\right)\left\|x_{n}-p\right\|^{2}+\left(\alpha_{n}+\hat{\beta}_{n}\right)\left\|t_{n}-p\right\|^{2} \\
\quad & \quad \hat{\beta}_{n}\left(\gamma_{n}\left\|t_{n}-p\right\|^{2}+c_{n}\right)-\left(\alpha_{n}-\kappa\right) \hat{\beta}_{n}\left\|t_{n}-S^{n} t_{n}\right\|^{2} \\
\leq & \left(1-\alpha_{n}-\hat{\beta}_{n}\right)\left\|x_{n}-p\right\|^{2}+\left(\alpha_{n}+\hat{\beta}_{n}\right)\left\|x_{n}-p\right\|^{2} \\
\quad & \quad-\left(\alpha_{n}-\kappa\right) \hat{\beta}_{n}\left\|t_{n}-S^{n} t_{n}\right\|^{2}+\beta_{n}\left(\gamma_{n}\left\|x_{n}-p\right\|^{2}+c_{n}\right) \\
= & \left\|x_{n}-p\right\|^{2}-\left(\alpha_{n}-\kappa\right) \hat{\beta}_{n}\left\|t_{n}-S^{n} t_{n}\right\|^{2}+\beta_{n}\left(\gamma_{n}\left\|x_{n}-p\right\|^{2}+c_{n}\right) .
\end{aligned}
$$


Further, it follows fron the definition of $\hat{\beta}_{n}$ that

$$
\begin{aligned}
\left\|z_{n}-p\right\|^{2} \leq & \left\|x_{n}-p\right\|^{2}-\left(\alpha_{n}-\kappa\right) \hat{\beta}_{n}\left\|t_{n}-S^{n} t_{n}\right\|^{2} \\
& +\beta_{n}\left(\frac{2 \gamma_{n}\left\|x_{n}-x_{1}\right\|^{2}+\left\|p-x_{1}\right\|^{2}+c_{n}}{1+\left\|x_{n}-x_{1}\right\|^{2}}\right) \\
\leq & \left\|x_{n}-p\right\|^{2}-\left(\alpha_{n}-\kappa\right) \hat{\beta}_{n}\left\|t_{n}-S^{n} t_{n}\right\|^{2}+\beta_{n}\left(2 \gamma_{n}\left(1+r_{0}^{2}\right)+c_{n}\right) \\
\leq & \left\|x_{n}-p\right\|^{2}-\left(\alpha_{n}-\kappa\right) \hat{\beta}_{n}\left\|t_{n}-S^{n} t_{n}\right\|^{2}+\theta_{n}
\end{aligned}
$$

where $\theta_{n}=\beta_{n}\left(2 \gamma_{n}\left(1+r_{0}^{2}\right)+c_{n}\right)$. Therefore, we have

$$
B_{r_{0}}\left(x_{1}\right) \cap F(S) \cap \Omega \subset C_{n}, \forall n \in \mathbb{N} .
$$

Step 2. We claim that the sequence $\left\{x_{n}\right\}$ converges strongly to an element in $C$, say $x^{*}$.

Since $\left\{C_{n}\right\}$ is a decreasing sequence of closed convex subset of $H$ such that $C^{*}=\cap_{n=0}^{\infty} C_{n}$ is a nonempty and closed convex subset of $H$, it follows from Lemma 2.6 that $\left\{x_{n+1}\right\}=\left\{P_{C_{n}} x_{1}\right\}$ converges strongly to $P_{C^{*}} x_{1}$, say $x^{*}$.

Step 3. We claim that $\lim _{n \rightarrow \infty} z_{n}=x^{*}$ and $\lim _{n \rightarrow \infty}\left\|t_{n}-S^{n} t_{n}\right\|=0$.

Indeed, the definition of $x_{n+1}$ shows that $x_{n+1} \in C_{n}$, i.e.,

$$
\left\|z_{n}-x_{n+1}\right\|^{2} \leq\left\|x_{n}-x_{n+1}\right\|^{2}-\left(\alpha_{n}-\kappa\right) \hat{\beta}_{n}\left\|t_{n}-S^{n} t_{n}\right\|^{2}+\theta_{n} .
$$

Note that $\gamma_{n} \rightarrow 0, c_{n} \rightarrow 0, x_{n} \rightarrow x^{*}$ as $n \rightarrow \infty$ and $\alpha_{n}>a>\kappa, \forall n \in \mathbb{N}$, we have $\theta_{n} \rightarrow$ $0,\left\|z_{n}-x_{n+1}\right\|^{2} \rightarrow 0,\left\|z_{n}-x_{n}\right\|^{2} \rightarrow 0$ and $z_{n} \rightarrow x^{*}$ as $n \rightarrow \infty$.

Further, it follows from (3.10) that

$$
(a-\kappa) \frac{b}{1+\left\|x_{n}-x_{1}\right\|^{2}}\left\|t_{n}-S^{n} t_{n}\right\|^{2} \leq\left\|x_{n}-x_{n+1}\right\|^{2}+\theta_{n} .
$$

Thus $\lim _{n \rightarrow \infty}\left\|t_{n}-S^{n} t_{n}\right\|=0$.

Step 4. We claim that $x^{*} \in F(S)$.

Since $z_{n}=\left(1-\alpha_{n}-\hat{\beta}_{n}\right) x_{n}+\alpha_{n} t_{n}+\hat{\beta}_{n} S^{n} t_{n}$, we have

$$
z_{n}-x_{n}=\left(\alpha_{n}+\hat{\beta}_{n}\right)\left(t_{n}-x_{n}\right)+\hat{\beta}_{n}\left(S^{n} t_{n}-t_{n}\right)
$$

Considering $0<a<\alpha_{n}+\hat{\beta}_{n}$, by Step 3 we have

$$
t_{n}-x_{n} \rightarrow 0, \quad t_{n} \rightarrow x^{*}, \text { as } n \rightarrow \infty \text {. }
$$

From (3.11), Lemma 2.5 and Step 3, we have

$$
\left\|S^{n} x^{*}-x^{*}\right\| \leq\left\|S^{n} x^{*}-S^{n} t_{n}\right\|+\left\|S^{n} t_{n}-t_{n}\right\|+\left\|t_{n}-x^{*}\right\| \rightarrow 0, \text { as } n \rightarrow \infty .
$$

Since $S: C \rightarrow C$ is uniformly continuous, by (3.12) we have

$$
S^{n+1} x^{*}=S\left(S^{n} x^{*}\right) \rightarrow S x^{*} \text {, as } n \rightarrow \infty .
$$

Hence, $S x^{*}=x^{*}$, i.e., $x^{*} \in F(S)$.

Step 5. We claim that $t_{n}-y_{n} \rightarrow 0, y_{n} \rightarrow x^{*}$, as $n \rightarrow \infty$.

By (3.5), for $p \in B_{r_{0}}\left(x_{1}\right) \cap F(S) \cap \Omega$, we have

$$
\left\|t_{n}-p\right\|^{2} \leq\left\|x_{n}-p\right\|^{2}+\left(\lambda_{n}^{2} L^{2}-1\right)\left\|x_{n}-y_{n}\right\|^{2}
$$


Therefore, we have

$$
\begin{aligned}
\left(1-\lambda_{n}^{2} L^{2}\right)\left\|x_{n}-y_{n}\right\|^{2} & \leq\left\|x_{n}-p\right\|^{2}-\left\|t_{n}-p\right\|^{2} \\
& \leq\left(\left\|x_{n}-p\right\|+\left\|t_{n}-p\right\|\right)\left(\left\|x_{n}-p\right\|-\left\|t_{n}-p\right\|\right) \\
& \leq\left(\left\|x_{n}-p\right\|+\left\|t_{n}-p\right\|\right)\left(\left\|x_{n}-t_{n}\right\|\right) .
\end{aligned}
$$

This together with (3.11) and $0<1-(1-a)^{2}<1-\lambda_{n}^{2} L^{2}$ implies that $\left\|x_{n}-y_{n}\right\| \rightarrow$ $0,\left\|t_{n}-y_{n}\right\| \rightarrow 0$ and $y_{n} \rightarrow x^{*}$, as $n \rightarrow \infty$.

Step 6. We claim that $x^{*} \in \Omega$.

Indeed, let

$$
T v= \begin{cases}A v+N_{C} v, & \text { if } v \in C, \\ \emptyset, & \text { if } v \notin C,\end{cases}
$$

where $N_{C} v$ be the normal cone to $C$ at $v \in C$. We have already mentioned in Section 2 that in this case $T$ is maximal monotone, and $0 \in T v$ if and only if $v \in \Omega$, see [18].

Let $(v, w) \in G(T)$, the graph of $T$. Then, we have $w \in T v=A v+N_{c} v$ and hence $w$ $A v \in N_{C} v$. So, we have

$$
\langle v-t, w-A v\rangle \geq 0, \forall t \in C .
$$

Noticing $t_{n}=P_{C}\left(x_{n}-\lambda_{n} A y_{n}\right)$ and $v \in C$, by (2.1) we have

$$
\left\langle x_{n}-\lambda_{n} A y_{n}-t_{n}, t_{n}-v\right\rangle \geq 0,
$$

and hence

$$
\left\langle v-t_{n}, \frac{t_{n}-x_{n}}{\lambda_{n}}+A y_{n}\right\rangle \geq 0 .
$$

From (3.13), (3.14) and $t_{n} \in C$, we have

$$
\begin{aligned}
\left\langle v-t_{n}, w\right\rangle & \geq\left\langle v-t_{n}, A v\right\rangle \\
& \geq\left\langle v-t_{n}, A v\right\rangle-\left\langle v-t_{n}, \frac{t_{n}-x_{n}}{\lambda_{n}}+A y_{n}\right\rangle \\
& \geq\left\langle v-t_{n}, A v-A t_{n}\right\rangle+\left\langle v-t_{n}, A t_{n}-A y_{n}\right\rangle-\left\langle v-t_{n}, \frac{t_{n}-x_{n}}{\lambda_{n}}\right\rangle
\end{aligned}
$$

Letting $n \rightarrow \infty$ in (3.15), considering $A: C \rightarrow H$ be a monotone, $L$-Lipschitz continuous mapping and $\left\{\lambda_{n}\right\} \subset(b / L,(1-a) / L)$, we have $\left\langle v-x^{*}, w\right\rangle \geq 0$. Since $T$ is maxmal monotone, we have $0 \in T x^{*}$ and hence $x^{*} \in \Omega=V I(C, A)$.

Step 7. We claim that the sequences $\left\{x_{n}\right\},\left\{y_{n}\right\}$, and $\left\{z_{n}\right\}$ converge strongly to $x^{*} \in F$ $(S) \cap \Omega$

From Step 4 and Step 6, we have $x^{*} \in F(S) \cap \Omega$. Therefore, it follows from Step 2, Step 3, and Step 5 that the sequences $\left\{x_{n}\right\},\left\{y_{n}\right\}$, and $\left\{z_{n}\right\}$ converge strongly to $x^{*} \in F(S)$ $\cap \Omega$. This completes the proof.

Remark 3.2. Theorem 3.1 improves and extends Theorems $C$ and D since

(1) the nonexpansive mapping $S$ in Theorem $C$ is extended to be an asymptotically $\kappa$-strict pseudocontractive mapping in the intermediate sense.

(2) the convergence condition that $\liminf _{n \rightarrow \infty}\left\langle A x_{n}, y-x_{n}\right\rangle \geq 0$ for all $y \in C$ in Theorem $\mathrm{D}$ is removed. 
(3) the boundedness assumptions that the intersection $F(S) \cap \Omega$ and the sequence $\left\{\Delta_{n}\right\}$ are both bounded in Theorem $\mathrm{D}$ are dispensed with.

(4) the requirement $(I-A)(C) \subset C$ in Theorem $\mathrm{D}$ is dropped off.

Corollary 3.3. Let $C$ be a nonempty closed convex subset of a real Hilbert space $H$. Let $S: C \rightarrow C$ be a uniformly continuous asymptotically $\kappa$-strict pseudocontractive mapping in the intermediate sense with sequence $\left\{\gamma_{n}\right\}$ such that $F(S)$ is nonempty. Let $\left\{x_{n}\right\}$ and $\left\{z_{n}\right\}$ be the sequences generated by the following algorithm with variable coefficients

$$
\left\{\begin{array}{l}
x_{1} \in C \quad \text { chosen arbitrary, } \\
z_{n}=\left(1-\alpha_{n}-\hat{\beta}_{n}\right) x_{n}+\alpha_{n} x_{n}+\hat{\beta}_{n} S^{n} x_{n} \\
C_{0}=C \\
C_{n}=\left\{z \in C_{n-1}:\left\|z_{n}-z\right\|^{2} \leq\left\|x_{n}-z\right\|^{2}-\left(\alpha_{n}-\kappa\right) \hat{\beta}_{n}\left\|x_{n}-S^{n} x_{n}\right\|^{2}+\theta_{n}\right\} \\
x_{n+1}=P_{C_{n}} x_{1}
\end{array}\right.
$$

for every $n \in \mathbb{N}, \quad$ where $\hat{\beta}_{n}=\frac{\beta_{n}}{1+\left\|x_{n}-x_{1}\right\|^{2}}, \quad \theta_{n}=\beta_{n}\left(2 \gamma_{n}\left(1+r_{0}^{2}\right)+c_{n}\right), \quad\left\{\alpha_{n}\right\} \subset(a, 1),\left\{\beta_{n}\right\} \subset(b, 1-a)$, and $\left\{\lambda_{n}\right\}$ $\subset(b, 1-a)$ for some $a \in(\kappa, 1)$ and some $b \in(0,1-a)$, and $c_{n}$ is as in (1.5), the positive real number $r_{0}$ is chosen so that $B_{r_{0}}\left(x_{1}\right) \cap F(S) \neq \emptyset$. Then the sequences $\left\{x_{n}\right\}$ and $\left\{z_{n}\right\}$ converge strongly to a point of $F(S)$.

Remark 3.4. Corollary 3.3 improves and extends [[4], Theorem 4.1] and Theorem D since the boundedness assumptions that the $F(S)$ and the sequence $\left\{\Delta_{n}\right\}$ are both bounded in [[4], Theorem 4.1] and Theorem D are dispensed with.

Recall that a mapping $T: C \rightarrow C$ is called pseudocontractive $[19,20]$ if

$$
\langle T x-T y, x-y\rangle \leq\|x-y\|^{2}, \forall x, y \in C \text {. }
$$

In fact, we see that (3.16) is equivalent to

$$
\|T x-T y\|^{2} \leq\|x-y\|^{2}+\|(I-T) x-(I-T) y\|^{2}, \quad \forall x, y \in C .
$$

Corollary 3.5. Let $C$ be a nonempty closed convex subset of a real Hilbert space $H$. Let $T: C \rightarrow C$ be a pseudocontractive, m-Lipschitz continuous mapping and let $S: C$ $\rightarrow C$ be a uniformly continuous asymptotically $\kappa$-strict pseudocontractive mapping in the intermediate sense with sequence $\left\{\gamma_{n}\right\}$ such that $F(S) \cap F(T)$ is nonempty. Let $\left\{x_{n}\right\}$, $\left\{y_{n}\right\}$, and $\left\{z_{n}\right\}$ be the sequences generated by the following algorithm with variable coefficients

$$
\left\{\begin{array}{l}
x_{1} \in C \quad \text { chosen arbitrary, } \\
y_{n}=\left(1-\lambda_{n}\right) x_{n}+\lambda_{n} T x_{n}, \\
t_{n}=P_{C}\left(x_{n}-\lambda_{n} y_{n}+\lambda_{n} T y_{n}\right), \\
z_{n}=\left(1-\alpha_{n}-\hat{\beta}_{n}\right) x_{n}+\alpha_{n} t_{n}+\hat{\beta}_{n} S^{n} t_{n}, \\
C_{0}=C \\
C_{n}=\left\{z \in C_{n-1}:\left\|z_{n}-z\right\|^{2} \leq\left\|x_{n}-z\right\|^{2}-\left(\alpha_{n}-\kappa\right) \hat{\beta}_{n}\left\|t_{n}-S^{n} t_{n}\right\|^{2}+\theta_{n}\right\}, \\
x_{n+1}=P_{C_{n}} x_{1},
\end{array}\right.
$$

$\begin{array}{llll}\text { for every } & n & \mathbb{N}, & \text { where }\end{array}$ $\hat{\beta}_{n}=\frac{\beta_{n}}{1+\left\|x_{n}-x_{1}\right\|^{2}}, \quad \theta_{n}=\beta_{n}\left(2 \gamma_{n}\left(1+r_{0}^{2}\right)+c_{n}\right), \quad\left\{\alpha_{n}\right\} \subset(a, 1),\left\{\beta_{n}\right\} \subset(b, 1-a)$ and $\left\{\lambda_{n}\right\}$ 
$\subset(b / L,(1-a) / L)$ for some $a \in(\kappa, 1)$ and some $b \in(0,1-a)$, and $c_{n}$ is as in (1.5), the positive real number $r_{0}$ is chosen so that $B_{r_{0}}\left(x_{1}\right) \cap F(S) \cap F(T) \neq \emptyset$. Then the sequences $\left\{x_{n}\right\},\left\{y_{n}\right\}$, and $\left\{z_{n}\right\}$ converge strongly to a point of $F(S) \cap F(T)$.

Proof. The following proof of this corollary is similar to that of [[10], Theorem 4.5].

Let $A=I-T$. It is easy to see that $y_{n}=P_{C}\left(x_{n}-\lambda_{n} A x_{n}\right)=\left(1-\lambda_{n}\right) x_{n}+\lambda_{n} T x_{n}$. Now let us show that the mapping $A$ is monotone and $(m+1)$-Lipschitz continuous. Indeed, observe that

$$
\langle A x-A y, x-y\rangle=\|x-y\|^{2}-\langle T x-T y, x-y\rangle \geq 0 .
$$

and

$$
\|A x-A y\|=\|x-y-(T x-T y)\| \leq(m+1)\|x-y\| .
$$

Next, let us show that $F(T)=\Omega=V I(C, A)$. Indeed, we have, for fixed $\lambda_{0} \in(0,1)$,

$$
T u=u \quad \Leftrightarrow \quad A u=0 \quad \Leftrightarrow \quad u=u-\lambda_{0} A u=P_{C}\left(u-\lambda_{0} A u\right) \quad \Leftrightarrow \quad\langle A u, y-u\rangle \geq 0, \forall y \in C .
$$

By Theorem 3.1, we obtain the desired conclusion. This completes the proof. $\square$

Remark 3.6. Theorem 3.5 improves and extends [[10], Theorem 4.5] since

(1) the convergence condition that $\liminf _{n \rightarrow \infty}\left\langle A x_{n}, y-x_{n}\right\rangle \geq 0$ for all $y \in C$ in [[10], Theorem 4.5] is removed.

(2) the boundedness assumptions that the intersection $F(S) \cap F(T)$ and the sequence $\left\{\Delta_{n}\right\}$ are both bounded in [[10], Theorem 4.5] are dispensed with.

By the careful analysis of the proof of Theorem 3.1, we can obtain the following result.

Theorem 3.7. Let $C$ be a nonempty closed convex subset of a real Hilbert space $H$. Let $A: C \rightarrow H$ be a monotone, L-Lipschitz continuous mapping and let $S: C \rightarrow C$ be a uniformly continuous asymptotically $\kappa$-strict pseudocontractive mapping in the intermediate sense with sequence $\left\{\gamma_{n}\right\}$ such that $F(S) \cap \Omega$ is nonempty and bounded. Let $\left\{x_{n}\right\},\left\{y_{n}\right\}$, and $\left\{z_{n}\right\}$ be the sequences generated by the following algorithm

$$
\left\{\begin{array}{l}
x_{1} \in C \text { chosen arbitrary, } \\
y_{n}=P_{C}\left(x_{n}-\lambda_{n} A x_{n}\right), \\
t_{n}=P_{C}\left(x_{n}-\lambda_{n} A y_{n}\right), \\
z_{n}=\left(1-\alpha_{n}-\beta_{n}\right) x_{n}+\alpha_{n} t_{n}+\beta_{n} S^{n} t_{n} \\
C_{0}=C \\
C_{n}=\left\{z \in C_{n-1}:\left\|z_{n}-z\right\|^{2} \leq\left\|x_{n}-z\right\|^{2}-\left(\alpha_{n}-\kappa\right) \beta_{n}\left\|t_{n}-S^{n} t_{n}\right\|^{2}+\theta_{n}\right\} \\
x_{n+1}=P_{C_{n}} x_{1}
\end{array}\right.
$$

for every $n \in \mathbb{N}, \quad$ where $\theta_{n}=\beta_{n}\left(\gamma_{n} \Delta_{n}+c_{n}\right), \Delta_{n}=\sup _{p \in F(S) \cap \Omega}\left\|x_{n}-p\right\|^{2},\left\{\alpha_{n}\right\} \subset(a, 1),\left\{\beta_{n}\right\} \subset(b, 1-a)_{a n d}\left\{\lambda_{n}\right\} \subset$ $(b / L,(1-a) / L)$ for some $a \in(\kappa, 1)$ and some $b \in(0,1-a)$, and $c_{n}$ is as in (1.5). Then the sequences $\left\{x_{n}\right\},\left\{y_{n}\right\}$ and $\left\{z_{n}\right\}$ converge strongly to a point of $F(S) \cap \Omega$.

Proof. Following the reasoning in the proof of Theorem 3.1, from (3.7), we take $\theta_{n}=$ $\beta_{n}\left(\gamma_{n} \Delta_{n}+c_{n}\right)$ and use $F(S) \cap \Omega$ instead of $B_{r_{0}}\left(x_{1}\right) \cap F(S) \cap \Omega$ in Step 1. From Step 2, we have the sequence $\left\{\Delta_{n}\right\}$ is bounded, and hence $\theta_{n}=\beta_{n}\left(\gamma_{n} \Delta_{n}+c_{n}\right) \rightarrow 0$ as $n \rightarrow \infty$. 
The remainder of the proof of Theorem 3.7 is similar to Theorem 3.1. The conclusion therefore follows. This completes the proof.

Theorem 3.8. Let $C$ be a nonempty closed convex subset of a real Hilbert space $H$. Let $A: C \rightarrow H$ be a monotone, L-Lipschitz continuous mapping and let $S: C \rightarrow C$ be a uniformly continuous asymptotically nonexpansive mapping in the intermediate sense such that $F(S) \cap \Omega$ is nonempty. Let $\left\{x_{n}\right\},\left\{y_{n}\right\}$, and $\left\{z_{n}\right\}$ be the sequences generated by the following algorithm

$$
\left\{\begin{array}{l}
x_{1} \in C \text { chosen arbitrary, } \\
y_{n}=P_{C}\left(x_{n}-\lambda_{n} A x_{n}\right), \\
t_{n}=P_{C}\left(x_{n}-\lambda_{n} A y_{n}\right) \\
z_{n}=\left(1-\alpha_{n}-\beta_{n}\right) x_{n}+\alpha_{n} t_{n}+\beta_{n} S^{n} t_{n} \\
C_{0}=C \\
C_{n}=\left\{z \in C_{n-1}:\left\|z_{n}-z\right\|^{2} \leq\left\|x_{n}-z\right\|^{2}-\alpha_{n} \beta_{n}\left\|t_{n}-S^{n} t_{n}\right\|^{2}+\theta_{n}\right\} \\
x_{n+1}=P_{C_{n}} x_{1},
\end{array}\right.
$$

for every $n \in \mathbb{N}$, where $\theta_{n}=\beta_{n} c_{n},\left\{\alpha_{n}\right\} \subset(a, 1),\left\{\beta_{n}\right\} \subset(b, 1-a)$, and $\left\{\lambda_{n}\right\} \subset(b / L,(1-$ $a) / L$ ) for some $a \in(0,1)$ and some $b \in(0,1-a)$, and $c_{n}$ is as in (1.2). Then the sequences $\left\{x_{n}\right\},\left\{y_{n}\right\}$, and $\left\{z_{n}\right\}$ converge strongly to a point of $F(S) \cap \Omega$.

Proof. In Theorem 3.1, whenever $S: C \rightarrow C$ is an asymptotically nonexpansive mapping in the intermediate sense, we have $\gamma_{n}=0, \kappa=0$ for all $n \in \mathbb{N}$. From (3.7), we have

$$
\left\|z_{n}-p\right\|^{2} \leq\left\|x_{n}-p\right\|^{2}-\left(\alpha_{n}-\kappa\right) \beta_{n}\left\|t_{n}-S^{n} t_{n}\right\|^{2}+\theta_{n}
$$

where $\theta_{n}=\beta_{n} c_{n}$. Thus, we have

$$
F(S) \cap \Omega \subset C_{n}, \forall n \in \mathbb{N},
$$

and hence, the result of Step 1 holds.

Next, following the reasoning in the proof of Theorem 3.1 and using $F(S) \cap \Omega$ instead of $B_{r_{0}}\left(x_{1}\right) \cap F(S) \cap \Omega$, we deduce the conclusion of Theorem 3.8. $\square$

Remark 3.9. Theorem 3.8 improves and extends Theorem $C$ since the nonexpansive mapping $S$ in Theorem $C$ is extended to be an asymptotically nonexpansive mapping in the intermediate sense.

\section{Acknowledgements}

The study was supported partly by the Natural Science Foundation of Anhui Province (11040606M01), the Natural Science Foundation of Anhui Educational Committee (KJ2011Z057), and the Specialized Research Fund 2010 for the Doctoral Program of Anhui University of Architecture.

\section{Competing interests}

The authors declare that they have no competing interests.

Received: 18 September 2011 Accepted: 26 April 2012 Published: 26 April 2012

\section{References}

1. Goebel, K, Kirk, WA: A fixed point theorem for asymptotically nonexpansive mappings. Proc Am Math Soc. 35(1):171-174 (1972). doi:10.1090/S0002-9939-1972-0298500-3

2. Bruck, RE, Kuczumow, T, Reich, S: Convergence of iterates of asymptotically nonexpansive mappings in Banach spaces with the uniform Opial property. Colloq Math. 65(2):169-179 (1993)

3. Liu, QH: Convergence theorems of the sequence of iterates for asymptotically demicontractive and hemicontractive mappings. Nolinear Anal TMA. 26(11):1835-1842 (1996). doi:10.1016/0362-546X(94)00351-H

4. Sahu, DR, Xu, HK, Yao, JC: Asymptotically strict pseudocontractive mappings in the intermediate sense. Nonlinear Anal TMA. 70(10):3502-3511 (2009). doi:10.1016/j.na.2008.07.007

5. Korpelevich, GM: An extragradient method for finding saddle points and for other problems. Ekonomika i Matematicheskie Metody. 12, 747-756 (1976) 
6. Takahashi, W, Toyoda, M: Weak convergence theorems for nonexpansive mappings and monotone mappings. J Optim Theory Appl. 118(2):417-428 (2003). doi:10.1023/A:1025407607560

7. Nadezhkina, N, Takahashi, W: Weak convergence theorem by an extragradient method for nonexpansive mappings and monotone mappings. J Optim Theory Appl. 128(1):191-201 (2006). doi:10.1007/s10957-005-7564-z

8. Zeng, LC, Yao, JC: Strong convergence theorem by an extragradient method for fixed point problems and variational inequality problems. Taiwan J Math. 10(5):1293-1303 (2006)

9. Nadezhkina, N, Takahashi, W: Strong convergence theorem by a hybrid method for nonexpansive mapping and Lipschitz-continuous monotone mappings. SIAM J Optim. 16(4):1230-1241 (2006). doi:10.1137/050624315

10. Ceng, LC, Yao, JC: Strong convergence theorems for variational inequalities and fixed point problems of asymptotically strict pseudocontractive mappings in the intermediate sense. Acta Appl Math. 115(2):167-191 (2011). doi:10.1007/ s10440-011-9614-x

11. Buong, N: Strong convergence theorem of an iterative method for variational inequalities and fixed point problems in Hilbert spaces. Appl Math Comput. 217(1):322-329 (2010). doi:10.1016/j.amc.2010.05.064

12. Ceng, LC, Petrusel, A, Lee, C, Wong, MM: Two extragradient approximation methods for variational inequalities and fixed point problems of strict pseudo-contractions. Taiwan J Math. 13(2A):607-632 (2009)

13. Ge, CS, Liang, J, Xiao, TJ: Iterative algorithms with variable coefficients for asymptotically strict pseudocontractions. Fixed Point Theory Appl 2010, 8 (2010). Article ID 948529

14. Marino, G, Xu, HK: Weak and strong convergence theorems for strict pseudocontractions in Hilbert spaces. J Math Anal Appl. 329(1):336-346 (2007). doi:10.1016/j.jmaa.2006.06.055

15. Matinez-Yanes, C, Xu, HK: Strong convergence of the CQ method for fixed point processes. Nolinear Anal TMA. 64(11):2400-2411 (2006). doi:10.1016/j.na.2005.08.018

16. Osilike, MO, lgbokwe, DI: Weak and strong convergence theorems for fixed points of pseudocontractions and solutions of monotone type operator equations. Comput Math Appl. 40, 559-567 (2000). doi:10.1016/50898-1221(00)00179-6

17. Ibaraki, T, Kimura, Y, Takahashi, W: Convergence theorems for generalized projections and maximal monotone operators in Banach spaces. Abstr Appl Anal. 2003(10):621-629 (2003). doi:10.1155/S1085337503207065

18. Rockafellar, RT: On the maximality of sums of nonlinear monotone operators. Trans Am Math Soc. 149(1):75-88 (1970). doi:10.1090/S0002-9947-1970-0282272-5

19. Takahashi, W: Nonlinear Function Analysis. Yokohama Publishers, Yokohama (2000)

20. Zhou, HY: Convergence theorems of fixed points for Lipschitz Pseudo-contractions in Hilbert spaces. J Math Anal Appl. 343(1):546-556 (2008). doi:10.1016/j.jmaa.2008.01.045

doi:10.1186/1687-1812-2012-68

Cite this article as: Ge: Strong convergence of iterative algorithms with variable coefficients for asymptotically strict pseudocontractive mappings in the intermediate sense and monotone mappings. Fixed Point Theory and Applications 2012 2012:68.

\section{Submit your manuscript to a SpringerOpen ${ }^{\circ}$ journal and benefit from:}

- Convenient online submission

- Rigorous peer review

- Immediate publication on acceptance

- Open access: articles freely available online

- High visibility within the field

- Retaining the copyright to your article

Submit your next manuscript at $\boldsymbol{s p r i n g e r o p e n . c o m ~}$ 